\title{
Emittance scans for CMS luminosity calibration
}

\author{
Olena Karacheban ${ }^{1 *}$, Peter Tsrunchev ${ }^{2}$ on behalf of CMS \\ ${ }^{1}$ CERN, Geneva, Switzerland \\ ${ }^{2}$ Sofia University, Sofia, Bulgaria
}

\begin{abstract}
Emittance scans are short van der Meer type scans performed at the beginning and at the end of LHC fills. The beams are scanned across each other in $x$ and $y$ planes. CMS has analyzed these scans to understand long-term trends in CMS luminosity detectors. The CMS Beam Radiation, Instrumentation and Luminosity (BRIL) project provides to LHC three independent online luminosity measurements from the Pixel Luminosity Telescope (PLT), the Fast Beam Condition Monitor (BCM1F) and the Forward Hadronic calorimeter (HF). The excellent performance of the BRIL detector front-ends, fast back-end electronics and CMS XDAQ based data processing and publication allow the use of emittance scans for linearity and stability studies of the luminometers. Emittance scans became a powerful tool and dramatically improved the understanding of the luminosity measurement during the year.
\end{abstract}

\section{$1 \mathrm{CMS}$ luminometers}

Any detector, which can measure particles hit rate and provide a linear dependency of the signal as a function of the luminosity, can be used as a luminometer. Multiple CMS luminometers are maintained by BRIL project, whose mission is to deliver luminosity and background measurements to CMS and LHC in real-time independent of the state of the CMS DAQ. A parallel BRIL DAQ infrastructure is used to achieve this task since 2015.

\subsection{Pixel Luminosity Telescope}

The PLT is silicon detector based on the similar technology to the phase-0 CMS pixel detector [1-2]. The PLT consists of 48 sensors, arranged into 16 telescopes ( 80 rows and 52 columns of pixels per sensor, with $150 \mathrm{~mm}$ wide and $100 \mathrm{~mm}$ high pixels). Each telescope makes an estimate of Poisson mean rate with the zero counting algorithm. Then the final uncalibrated PLT rate is set as the average of Poisson mean rates of available telescopes.

PLT and BCM1F are installed on both ends of CMS about $1.8 \mathrm{~m}$ away from the interaction point in the tracker volume. They are sharing one carriage surrounding the beam pipe. PLT has an active cooling system.

\subsection{Fast Beam Condition Monitor}

\footnotetext{
* Corresponding author: olena.karacheban@,cern.ch
} 
The BCM1F is dedicated detector for luminosity and machine induced background (MIB) measurement [2]. Three types of sensors are employed: poly-crystalline diamond (pCVD, active area of one pad is about $6.1 \mathrm{~mm}^{2}$ ), silicon ( $\mathrm{Si}$, sensor size of about $6 \mathrm{~mm}^{2}$ ) and single-crystalline diamond ( $\mathrm{SCVD}$, active area of one pad is about $10.3 \mathrm{~mm}^{2}$ ). Particle hit rate counting is used for luminosity measurement. Data from each sensor type is processed separately, providing three independent luminosity measurements. For BCM1F diamond sensors cooling is not required and silicon sensors are relying on the indirect ambient cooling from PLT and the dry volume of the inner tracker.

\subsection{Hadronic Forward Calorimeter}

The HF is the most senior luminometer of CMS, which was used from the first running period of the LHC. During LHC long shutdown 1 the back-end readout was significantly upgraded. This allowed for more robust readout during central CMS DAQ failures and added the option to readout multiple rate algorithms simultaneously. The primary occupancy algorithm (HFOC) based on the counting of the HF towers with the energy deposition higher than defined threshold was complemented starting from 2017 by the new algorithm (HFET), based on the sum of the transverse energy counting [2,3].

\section{Calibration of the luminometer}

For the calibration of the luminometer special experimental conditions are created - Van der Meer (VdM) scans - a method originally proposed by Simon van der Meer and first exploited at the CERN ISR [4].

\subsection{Van der Meer scans}

The series of VdM scans is performed once per year in the dedicated LHC fill. Only well separated bunches with about $9 \times 10^{10}$ protons per beam are colliding (no long range interactions). Special beam optics is required in this fill to provide much wider beams than in normal LHC operation (beam overlap region about 100-130 $\mu \mathrm{m}, \beta^{*}=1917 \mathrm{~cm}$, zero crossing angle).
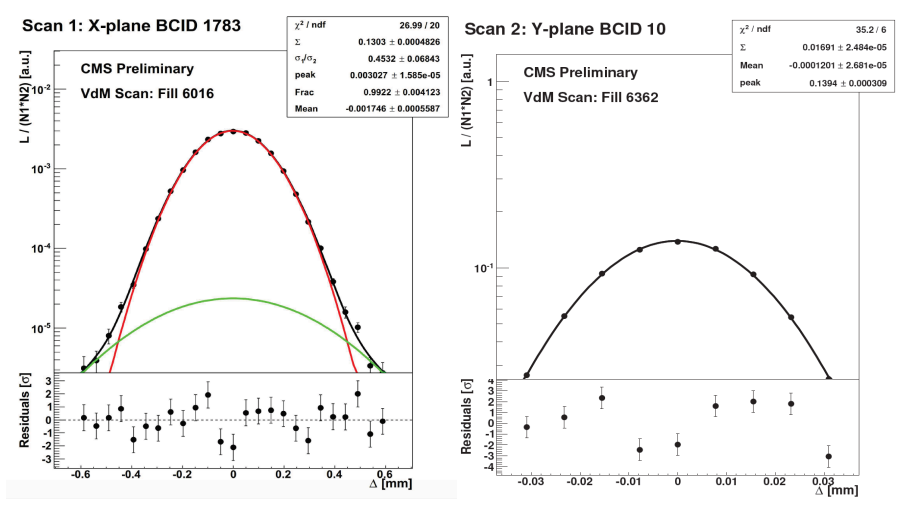

Fig. 1. An example of the VdM scan shape with double Gaussian function fit (left) and emittance scans shape with single Gaussian function fit (right).

The standard VdM method consists of scanning colliding bunches across each other in the plane transverse to the beam axis in order to determine their overlap region. The two 
beams are moved with respect to each other in steps (displacement steps) in the $x$ and then in the $y$ plane, and the rate $R$ is measured by the luminometers. The maximum count rate is reached when beams are colliding with zero displacement, "head-on". Bunches have a Gaussian-function-like shape. An example of the VdM shape measured by the PLT detector is shown in Fig. 1 (left). Black dots correspond to the measured count rate normalized by bunch current at each separation step, green and red curves are two Gaussian functions and black curve correspond to the resulting fit. The residuals of the fit in units of the statistical errors of the measured rates are also shown in Fig. 1 at the bottom. The peak rate in $x$ scan $\left(R_{\max , x}\right)$ and in $y$ scan $\left(R_{\max , y}\right)$, peak position, and beam overlap width in $x$ scan $\left(\Sigma_{x}\right)$ and in $y$ scan $\left(\Sigma_{y}\right)$ are obtained from the fit and used to calculate the visible cross-section also called sigma visible (SigmaVis, $\sigma_{v i s}$ ), as shown in Eq.1.

$$
\sigma_{v i s}=\pi \Sigma_{x} \Sigma_{y}\left(R_{\max , x}+R_{\max , y}\right)
$$

$\sigma_{\text {vis }}$ values obtained in the $\mathrm{VdM}$ scans program are used for absolute luminosity calibration, as shown in Eq.2. LHC orbit frequency $f_{L H C}=11.25 \mathrm{kHz}$.

$$
L=R f_{L H C} / \sigma_{v i s}
$$

For the detailed study of the non-factorizability of the beams, which is the main source of the systematic uncertainty of the VdM method, additional scans are performed: imaging scans for xy-correlations study and length scale calibration for nominal beam separation correction. The VdM program and systematic uncertainties are described in detail in [3].

\subsection{Emittance scans}

During physics data taking to maximize luminosity the LHC machine is filled with more than 2500 bunches with about $12 \times 10^{10}$ protons per bunch, focused using beam optics close to interaction point $\left(\beta^{*}=30 \mathrm{~cm}\right)$ and reaching beam overlap region of about $20 \mu \mathrm{m}$. Bunches are colliding with crossing angle in the range 130-150 $\mu \mathrm{rad}$.
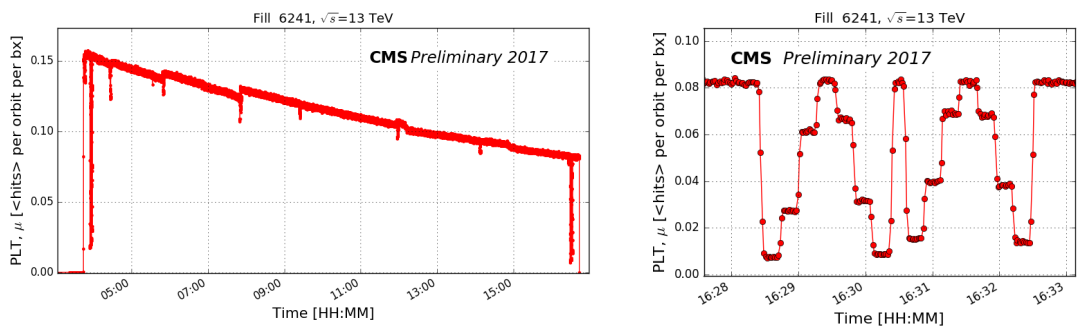

Fig. 2. $\mu$ values for the PLT during the whole fill 6241 (left) and zoom into the period of emittance scan towards the end of the fill (right).

Fig.2 (left) illustrates the mean value of the number of hits per orbit per bunch crossing ( $\mu$ values) for the PLT during fill 6241. An almost 50\% drop in luminosity is observed after 12 hours of collisions. In 2017 so-called "emittance scans" were performed at the beginning and at the end of the fill for luminosity calibration purposes. In Fig.2 (left) they are seen as two deep dips, one at the beginning ("early" emittance scan) and one at the end of the fill ("late" emittance scan). The smaller dips along the fill correspond to beam optimizations, which are performed to find the position where beams collide head-on or after a crossing angle change. In Fig.2 (right), a zoom into the late emittance scan is shown. Scans of the beam in the $x$ and $y$ directions are performed with 7 steps each, staying 10 seconds on each separation step. After the scan in $x$ the beams are brought to head-on position for approximately 5 seconds (step to the maximum rate between the scans) after 
which the scan in $y$ plane is performed. Later in the year emittance scans with 9 steps were performed for better fit quality.

\section{Stability and linearity measurement of the CMS luminometers}

As a part of a precise luminosity measurement for physics analysis, a measurement of the stability of each luminometer and the linearity analysis are of critical importance. For the particular energy of the LHC beams the measured $\sigma_{v i s}$, as a characteristic of the detector, is expected to be constant, unless inefficiency or nonlinearity occur. The estimates of stability and linearity based on emittance scans and per bunch $\sigma_{\text {vis }}$ measurement are powerful diagnostic tools and found the use to derive corrections on large datasets.

\subsection{Per bunch crossing measurement of the $\sigma_{v i s}$ and emittance}

Two independent analyses of emittance scans are launched: a Python-based framework (FW) and an XDAQ-based application. The results are published on the monitoring webpages in real-time for the XDAQ-based analysis and typically within 15 minutes for the Python-based framework, which has however the advantage of being rerunnable.
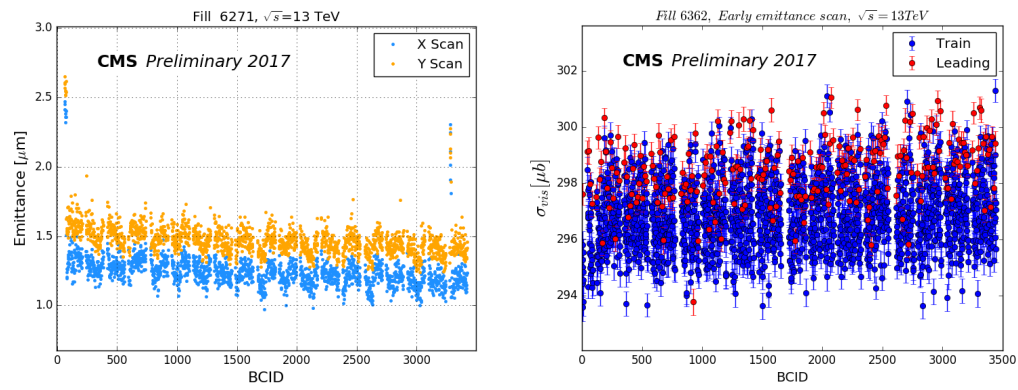

Fig. 3. Left: per bunch crossing emittance values calculated based on beam width in $x$ and $y$ emittance scans. Fill 6271, PLT data. Right: visible cross-section as a function of Bunch Crossing number (BCID) for leading and train bunches. Fill 6362, PLT data from an early emittance scan is shown.

Due to different emittance values, a single LHC fill contains bunches of varying Single Bunch Instantaneous Luminosity (SBIL). The peak rate values obtained from fits of the emittance scan shapes are used to calculate the SBIL per bunch crossing. The usual range of the SBIL at the beginning of the fill is between 6 and $10 \mathrm{~Hz} / \mu \mathrm{b}$, while the SBIL range for the late scan strongly depends on the length of the fill. The beam overlap region obtained from the fits of the emittance scan shapes is used to calculate per bunch emittance values in $x$ and in $y$. An example of the emittance values obtained in fill 6271 is shown in Fig.3 (left). The presence of the crossing angle in $x$ plane and longitudinal profile of the beam are taken into account. As the bunch length is measured as a full width half maximum of the longitudinal profile of the bunch and is overestimated in the case of non-Gaussian beam, a correction in size of $0.5 \mu \mathrm{m}$ is applied to emittance in $x$ [5].

Per bunch crossing $\sigma_{\text {vis }}$ measured in one early emittance scan for PLT detector are shown in Fig.3 (right) separately for leading and train bunches. The same methodology is used for all BRIL luminometers. The difference of $\sigma_{\text {vis }}$ between leading and train bunches increases towards the end of the fill due to nonlinearity, as discussed in the section 3.2.

\subsection{Stability and linearity measurement based on the emittance scans}

The $\sigma_{\text {vis }}$ obtained in all early emittance scans performed in 2017 operation, as shown in Fig.4 (left), were used to monitor the efficiency of the PLT detector. The downward trends 
highlighted in pink show decreases in efficiency, which were mitigated by an increase of the operational high voltage (HV). The time of the HV change are indicated with black lines. The grey vertical line points to the time of the filling scheme change from long bunch trains to a " 8 bunches filled 4 bunches empty" ( $8 \mathrm{~b} 4 \mathrm{e})$ scheme.
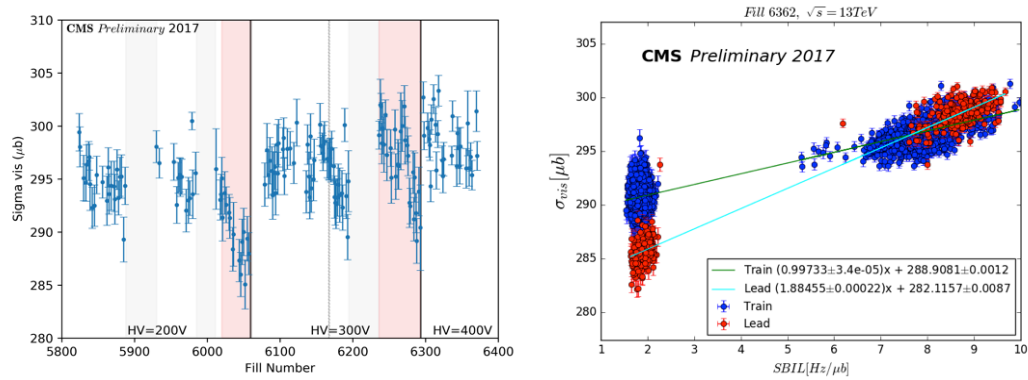

Fig. 4. PLT data is shown. Left: $\sigma_{\text {vis }}$ as a function of fill number. Right: $\sigma_{\text {vis }}$ as a function of SBIL used for nonlinearity monitoring for leading and train bunches.

A nonlinearity measurement is done for every fill based on $\sigma_{\text {vis }}$ vs. SBIL plots. An example of linear fits of the $\sigma_{\text {vis }}$ as a function of SBIL is shown in Fig. 4 (right) for leading and train bunches in red and blue respectively. One point of the plot corresponds to one of 3564 BCIDs. For PLT a final nonlinearity correction measured in the period of stability is applied for leading and train bunches separately taking into account the fraction of each type in a particular fill. For BCM1F and HF correction is estimated for leading and train bunches separately, however a single nonlinearity correction is applied in final calibrations.

The analysis of emittance scans as VdM scans through 2017 data-taking provides estimates of linearity and time-dependent detector efficiency. These estimates were used to correct the linearity and inefficiency per detector independently. These are the first results using this methodology.

\section{Acknowledgements}

We are thankful to LHC colleagues, in particular M. Hostettler and J. Wenninger, for useful discussions and performing the scans. Big thank goes to our supervisors A.Dabrowski, D.Stickland, W.Lohmann and colleagues Z.Xie, J.Daugalas, A.Delannoy, M.Guthoff, P.Lujan, C.Palmer and A.Kornmayer for detectors operations and help with data analysis.

\section{References}

1. M. Barbero et al., Design and test of the CMS pixel readout chip, Nucl. Instrum. Methods Phys. Res., A 517 (2004) 349

2. M. Guthoff on behalf of the CMS, Instrumentation for beam radiation and luminosity measurement in the CMS experiment using novel detector technologies, Nucl. Instrum. Methods Phys. Res., A 845 (2017) 1-7

3. CMS Collaboration, CMS Luminosity Measurement for the 2017 Data Taking Period, CMS PAS LUM-17-004 (2018), https://cds.cern.ch/record/2621960

4. S. van der Meer, Calibration of the Effective Beam Height in the ISR, CERN-ISR-PO68-31 (1968)

5. M. Hostettler et al., Luminosity Scans for Beam Diagnostics, submitted to Physical Review ST Accelerators and Beams, pre-print https://arxiv.org/abs/1804.10099 